\title{
Il est bon que le débat ait lieu sur une question complexe
}

Jean Martin

jean.martin[at]saez.ch
Un grand merci à chacun(e) des collègues qui ont pris la peine de donner leur opinion suite à mon article. D’abord, que je précise que je ne prétends jamais avoir forcément raison. Par des contacts de divers côtés (y compris des réactions à mon article qui n'ont pas donné lieu à des lettres de lecteur), je constate ne pas être le seul à avoir des préoccupations à propos des «boîtes à bébé», mais les opinions différentes qui sont exprimées ont mon respect - et, en réalité, nous sommes d'accord sur beaucoup de points.

Entre autres, la réaction de notre confrère Rupp est appréciée, tant par sa parfaite courtoisie que par sa substance. Il est clair que la situation de femmes tentées d'abandonner un nouveau-né est toujours complexe, et certaines attitudes stigmatisantes/rejetantes qui persistent dans notre société n'arrangent pas les choses. Un tel abandon, qu'il soit anonyme ou que la mère le donne en vue d'adoption, est «kein einfacher Weg», c'est certain. Il importe de considé- rer les enjeux qui portent alors sur deux existences, celle du bébé et celle de la mère, qui doivent toutes deux retenir notre attention, et bénéficier du soutien de professionnels et institutions compétents. Tout à fait d'accord avec le Dr Bättig qu'est essentielle une «Total-Mitmenschlichkeit».

Où je continue à diverger avec certains des correspondants, c'est sur l'importance d'avoir accès à des indications sur son origine (avec beaucoup d'autres, dont le Dr Jungi et le Dr Schär, je ne crois pas du tout qu'il s'agisse d'un "postulat éthique fictif»...), et sur la désirabilité de l'installation de plus (beaucoup plus?!) de boîtes à bébé dans notre pays.

Dans tous les cas, il est bon qu'un débat large ait lieu, par le canal judicieux de notre journal professionnel. On doit souhaiter que les points discutés dans cet échange fassent l'objet de réflexions complémentaires des responsables médico-sanitaires concernés, des autorités civiles et du public en général. 